\title{
Multi-band functional tunable LH impedance transformer
}

DOI:

10.1163/156939309787604652

Link to publication record in Manchester Research Explorer

\section{Citation for published version (APA):}

Abdalla, M. A., \& Hu, Z. (2009). Multi-band functional tunable LH impedance transformer. Journal of Electromagnetic Waves and Applications, 23(1), 39-47. https://doi.org/10.1163/156939309787604652

\section{Published in:}

Journal of Electromagnetic Waves and Applications

\section{Citing this paper}

Please note that where the full-text provided on Manchester Research Explorer is the Author Accepted Manuscript or Proof version this may differ from the final Published version. If citing, it is advised that you check and use the publisher's definitive version.

\section{General rights}

Copyright and moral rights for the publications made accessible in the Research Explorer are retained by the authors and/or other copyright owners and it is a condition of accessing publications that users recognise and abide by the legal requirements associated with these rights.

\section{Takedown policy}

If you believe that this document breaches copyright please refer to the University of Manchester's Takedown Procedures [http://man.ac.uk/04Y6Bo] or contact uml.scholarlycommunications@manchester.ac.uk providing relevant details, so we can investigate your claim.

\section{OPEN ACCESS}




\title{
MULTI-BAND FUNCTIONAL TUNABLE LH IMPEDANCE TRANSFORMER
}

\author{
M. A. Abdalla and Z. Hu \\ MACS Group \\ School of EEE \\ University of Manchester \\ Manchester M60 1QD, UK
}

Abstract-This paper presents, for the first time, the analysis and design performance of tunable left handed coplanar waveguide transformer designed on a ferrite substrate. The proposed transformer is studied analytically and verified numerically. Results show that the proposed transformer has tunable dual band of operation with frequency tuning range from $2.35 \mathrm{GHz}$ to $3.85 \mathrm{GHz}$ with return loss from $10 \mathrm{~dB}$ to $25 \mathrm{~dB}$. Such tunability can be achieved by changing the DC magnetic bias. The advantages of the proposed transformer are its tuning capability, its multi functionality operation, and its compact size.

\section{INTRODUCTION}

Left handed (LH) metamaterials are artificially constructed materials having electromagnetic properties that do not exist in nature. LH metamaterials, which were originally investigated and named by Veselago in 1967 [1], have simultaneously negative permittivity and permeability.

The LH metamaterials have been realized either as a volume version or a planar version based on the use of split ring resonators (SRRs) with a wire array placed in space, or transmission line loaded with complementary SRR (CSRR)/capacitive gap pairs $[2,3]$. A transmission line (TL) periodically loaded with series capacitors and shunt inductors can also exhibit left handed properties [4,5]. Planar left handed metamaterials have been realized in different models of left handed coplanar waveguide (CPW) [6,7]. Many novel planar

Corresponding author: M. A. Abdalla (mahmoud.abdalla@postgrad.manchester.ac.uk). 
microwave components LH elements have been introduced making use of the unique properties of the left handed structures $[8,9]$.

Ferrite medium has nonreciprocal and tunable properties [10] due to its dispersive permeability. Therefore, a tunable and nonreciprocal LH TL is expected using ferrite substrate. Such features have been demonstrated by Tsutsumi $[11,12]$ using a microstrip TL over mixed ferrite and dielectric substrate and Abdalla $[13,14]$ using CPW TL on a pure ferrite substrate which can be applied in many microwave applications [15].

In this paper, we propose a multi-band functional tunable and compact quarter wavelength transformer implemented using a $\mathrm{LH}$ TL on ferrite substrate in CPW geometry structure. The LH TL is designed using loading series air gap capacitive load and shunt meandered line inductive load. The proposed transformer has tunable dual band of operation with frequency tuning range from $2.35 \mathrm{GHz}$ to $3.85 \mathrm{GHz}$. The proposed transformer is studied analytically and its performance has been verified numerically. The proposed tunable transformer has the advantages of its compact size and its multi band functionality, in addition to its lower DC magnetic bias compared to microstrip TL because of its smaller demagnetization factor.

\section{THEORY}

The electrical length of a conventional transmission line is linearly proportional to the frequency. The conventional transformer is a quarter wavelength transmission line, $l=\lambda / 4$, such that it achieves an electrical length of $\Phi=-\pi / 2$ at the operating frequency $\left(f_{1}\right)$. The transmission line transformer can achieve an equivalent electrical length of $\Phi=-n(\pi / 2)$ where $n$ is an odd integer positive number. These electrical lengths are satisfied at frequencies represent the odd harmonics of the first frequency $\left(f_{1}\right)$, i.e., $f_{n}=n * f_{1}$.

Practical LH TL is a combination of both the left handedness due to the effect of the loading elements and right handedness due to the parasitic elements of the hosting transmission line. Thus it is called composite right/left handed (CRLH) TL [8]. As a result, a CRLH TL can achieve arbitrary designed positive and negative electrical lengths according to the loaded LH elements and right handed $(\mathrm{RH})$ parasitic elements. Therefore a CRLH can achieve the same condition of the $\pi / 2$ phase shift or equivalent at arbitrary frequencies which is not a condition to be the odd harmonics of the first resonant point. The choice of theses arbitrary frequencies depends on the design of the cut off frequencies of both $\mathrm{LH}$ and $\mathrm{RH}$ passbands such that any designed frequency must fall within any passband. 
Novel LH transformer can be designed as a novel application of the CRLH theory $[8,9]$. In practice, it is implemented by cascading a practical LH TL, CRLH TL, with a short RH TL section for feeding purposes. The principles of operations of a multifunctional LH TL transformer is based on making use of the nonlinear phase properties of LH TL such that it has $\pi / 2$ phase shift, or equivalent, at more arbitrary operating frequencies which are in either LH or $\mathrm{RH}$ passband. Along with phase shift condition satisfaction, the LH TL characteristic impedance should be constant at these different frequencies. Such characteristic impedance should be designed similar to conventional quarter wavelength transformer as

$$
Z_{o l}=Z_{C R L H} \frac{Z_{L o a d}+Z_{C R L H} \tan \left(\beta_{C R L H} l\right)}{Z_{C R L H}+Z_{L o a d} \tan \left(\beta_{C R L H} l\right)}
$$

where $Z_{C R L H}$ is the characteristic impedance of the CRLH TL, ${ }^{\beta} C R L H$ is the propagation constant of the CRLH TL, $Z_{\text {Load }}$ is the load impedance, and $Z_{o l}$ is the line characteristic impedance.

The phase shift of a CRLH transmission line of length $(l)$ can be approximated as given in [8],

$$
\phi_{C L R H}=-\beta_{C R L H} l=\left(\frac{1}{\omega \sqrt{C_{L}^{\prime} L_{L}^{\prime}}}-\omega \sqrt{C_{E}^{\prime} L_{R}^{\prime}}\right) l
$$

The characteristic impedance of a CRLH transmission line is given as $[8]$

$$
Z_{o C L R H}=Z_{L} \sqrt{\frac{\left(\omega / \omega_{s e}\right)^{2}-1}{\left(\omega / \omega_{s h}\right)^{2}-1}}
$$

where $Z_{L}$ is the characteristic impedance of the ideal left handed transmission line, $\omega_{s e}$ and $\omega_{s h}$ represent the resonance frequency for the series and shunt branches of the transmission line equivalent circuit respectively. All these parameters can be expressed as follow

$$
\begin{gathered}
\omega_{s e}=\frac{1}{\sqrt{L_{R}^{\prime} C_{L}^{\prime}}}, \quad \omega_{s h}=\frac{1}{\sqrt{L_{L}^{\prime} C_{R}^{\prime}}} \\
Z_{L}=\sqrt{\frac{L_{L}^{\prime}}{C_{L}^{\prime}}}
\end{gathered}
$$

where $L_{R}^{\prime}$, and $C_{R}^{\prime}$ are the per unit length parasitic inductance and capacitance, while $L^{\prime} L$, and $C^{\prime} L$ represent the product of the loading 
inductance and capacitance with the left handed section length. It can be shown that at low frequencies, this characteristic impedance is reduced to the left handed impedance $\left(Z_{L}\right)$.

The progressive phase shift and the characteristic impedance of a CRLH transmission line of length $(l)$ implemented on ferrite hosting transmission line can be redefined in terms of its medium parameters, the ferrite relative permittivity $\varepsilon_{f}$ and the relative permeability $\mu_{f}$, as illustrated in $[13,14]$ to be as follow:

$$
\begin{gathered}
\phi_{f}=\omega l \sqrt{\left(\mu_{o} \mu_{f}-\frac{1}{\omega^{2} C_{L}^{\prime}}\right)\left(\varepsilon_{o} \varepsilon_{f}-\frac{1}{\omega^{2} L_{L}^{\prime}}\right)} \\
Z_{o f C L R H}=Z_{L} \sqrt{\frac{1-\omega^{2} \mu_{o} \mu_{f} C_{L}^{\prime}}{1-\omega^{2} \varepsilon_{o} \varepsilon_{f} L_{L}^{\prime}}}
\end{gathered}
$$

The hosting ferrite TL has a dispersive permeability according to the applied DC magnetic bias which causes its electrical parameters to be dispersive. Thus, it is expected to achieve tunable performance of the CRLH TL when implemented on the ferrite substrate by applying different DC magnetic bias values.

\section{STRUCTURE}

The layout of the proposed LH CPW transformer on ferrite substrate is shown in Figure 1(a) which shows that the transformer consists of two unit CRLH cells implemented using a series air gap capacitor and a shunt meandered line inductor. The detailed sketch for the loading shunt meandered line inductor is shown in Figure 1(b). The dimensions of the loading elements are chosen to satisfy the desired impedance in the LH passband as explained later. The employed ferrite substrate is the commercial Trans Tech G-113* with thickness $1 \mathrm{~mm}$. Its electric and magnetic material parameters are, dielectric constant $\varepsilon_{f}=15$, dielectric loss tangent $<0.0002$, saturation magnetization $4 \pi M_{S}=1780$ Gauss, and magnetic linewidth $\Delta H_{0} \leq 25$ Oe. An internal DC magnetic field $\left(H_{0}\right)$ is applied to the ferrite substrate in the direction shown in Figure 1(a), causing the ferrite substrate to have the saturation magnetization in the same direction. The ferrite dispersive relative permeability can be expressed as [13]

$$
\mu_{f}=\frac{\left(\mu_{o} \gamma\right)^{2} H_{0}\left(H_{0}+M_{s}\right)-\omega^{2}}{\left(\mu_{o} \gamma H_{0}\right)^{2}-\omega^{2}}
$$

where $\gamma$ is the gyromagnetic ratio of the ferrite and $\mu_{o}$ is the free space permeability. 


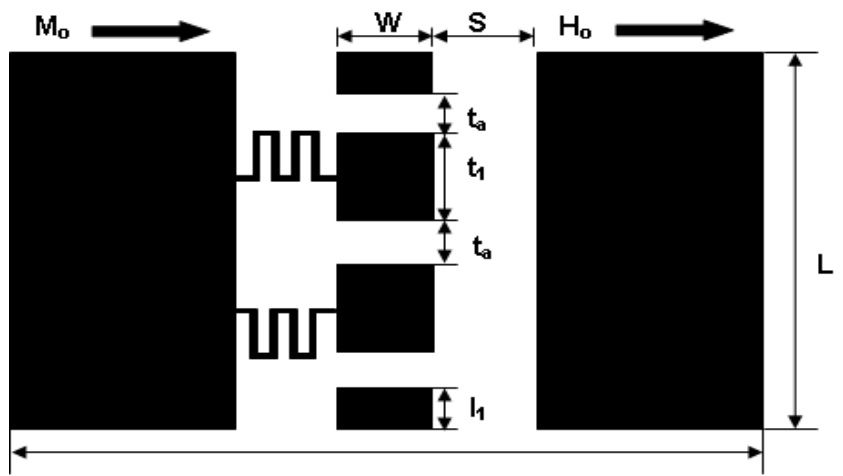

a

(a)

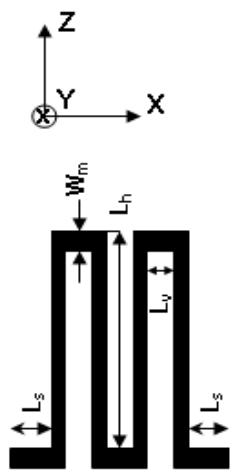

(b)

Figure 1. (a) The layout geometry of the ferrite LH CPW transformer $a=35 \mathrm{~mm}, L=6.52 \mathrm{~mm}, W=1.8 \mathrm{~mm}, t_{1}=2 \mathrm{~mm}, t_{a}=0.5 \mathrm{~mm}$, $l_{1}=0.5 \mathrm{~mm}$; (b) The detailed geometry of meandered line inductor $W_{m}=0.05 \mathrm{~mm}, L_{s}=0.45 \mathrm{~mm}, L_{h}=0.95 \mathrm{~mm}, L_{v}=0.5 \mathrm{~mm}$.

The design of the proposed transformer is first done assuming a very high DC magnetic bias is applied to the ferrite substrate. At that bias, the dispersive properties of the ferrite substrate will be shifted to high frequency which means that the ferrite substrate is characterized by only its isotropic properties at the operating frequency band of interest. At this condition, the proposed transformer is designed to match perfectly a $150 \Omega$ load to $50 \Omega$ line at two arbitrary centre frequencies selected to be $2.7 \mathrm{GHz}$ and $3.8 \mathrm{GHz}$.

To meet these design requirements, first, the CPW TL is designed to achieve $90^{\circ}$ phase shift along its output terminal at both $2.7 \mathrm{GHz}$ and $3.8 \mathrm{GHz}$. This was achieved by the proper selection of the loading elements and parasitic elements such that their contribution in (2) introduces $\pi / 2$ phase shift, or equivalent, at the aforementioned two operating frequencies. Second, the CPW LH TL characteristic impedance was calculated to be $86 \Omega$ at the two operating frequencies, calculated from (1) to match $150 \Omega$ load to a $50 \Omega$ line with $\pi / 2$ phase shift. This also was fulfilled by the aid of the loading and parasitic elements such that their contributions again in (3), simplified in (5) at lower operating frequencies as explained before, satisfy the desired characteristic impedance. Next, a numerical optimization was applied for the sake of the exact simultaneous satisfaction of the phase condition and the characteristic impedance condition at the two different operating frequencies. 


\section{NUMERICAL RESULTS}

To verify these analytical results, the proposed transformer has been analyzed using full wave simulation. The commercial software ANSOFT-HFSS is employed. For simplicity, the applied DC magnetic field is assumed to be uniform in all studied cases.

The phase shift of the transmission scattering parameter $\left(S_{21}\right)$ of the proposed ferrite LH TL for $H_{0}=50000$ Oe is shown in Figure 2 using the right vertical axis. As shown in the figure, that the TL has a $-90^{\circ}$ phase shift, equivalent to $270^{\circ}$, between $2.65 \mathrm{GHz}$ and $2.7 \mathrm{GHz}$ and it has $+90^{\circ}$ phase shift between $3.8 \mathrm{GHz}$ and $3.85 \mathrm{GHz}$.

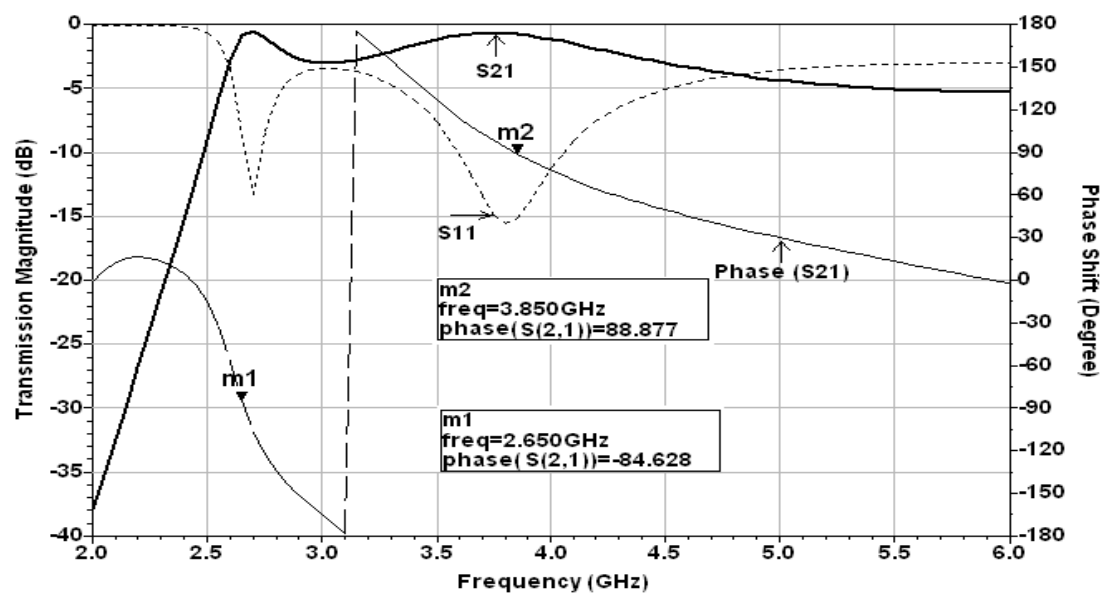

Figure 2. The full wave scattering parameters (magnitude and phase) of the dual band ferrite transformer for $H_{0}=50,000$ Oe.

Then transmission scattering parameters of proposed $\mathrm{LH}$ transformer connecting a $50 \Omega$ line to a terminating load of $150 \Omega$ for the same DC magnetic bias are shown in the same figure using the left vertical axis. It is obvious that the proposed transformer matches the load to the line with minimum return loss better than $16 \mathrm{~dB}$ at $3.8 \mathrm{GHz}$, the primary operating frequency, and better than $10 \mathrm{~dB}$ close to $2.7 \mathrm{GHz}$, the secondary operating frequency. It is clear that these two frequencies are identical to those frequencies around which the used LH TL illustrates $90^{\circ}$ phase shift at its output terminals. In both cases, the transmission coefficient is close to $0 \mathrm{~dB}$. The proposed transformer illustrates dual operating bands around its primary and secondary center operating frequencies. The primary band has reasonable bandwidth that extends approximately from 
$3.25 \mathrm{GHz}$ to $4.75 \mathrm{GHz}$. On the other hand, the secondary band has a narrow bandwidth due to the fast variation of the phase of $\left(S_{21}\right)$ in the right hand axis around the secondary operating frequency. A narrow overlap between the two separated bands that results on a narrow non perfect matching bandwidth between $3 \mathrm{GHz}$ and $3.25 \mathrm{GHz}$.

The tuning capability of the proposed left handed transformer can be illustrated numerically for different lower DC magnetic bias values of 1500 and 2000 Oe as shown in Figure 3. It can be seen that the proposed transformer still have dual band operation. The secondary frequency is tuned from $2.4 \mathrm{GHz}$ to $2.85 \mathrm{GHz}$ while the primary operating frequency is tuned from $3.3 \mathrm{GHz}$ to $3.85 \mathrm{GHz}$ for $H_{0}=2000$ Oe and 1500 Oe respectively.

For further studied cases of the applied DC bias from 1000 Oe to $5000 \mathrm{Oe}$, the two perfect matching frequencies illustrate a non linear variation with the applied DC magnetic bias as shown in Figure 4. It can be seen that both of them increase by increasing $H_{0}$ from 1000 Oe until 1500 Oe then they are almost constant by increasing $H_{0}$ to 1750 Oe before they decrease by more increasing of $H_{0}$ till 2000 Oe. Then, by further increasing of $H_{0}$, both frequencies start to increase slightly and approach the designed values assuming high DC magnetic bias is applied shown in Figure 2.

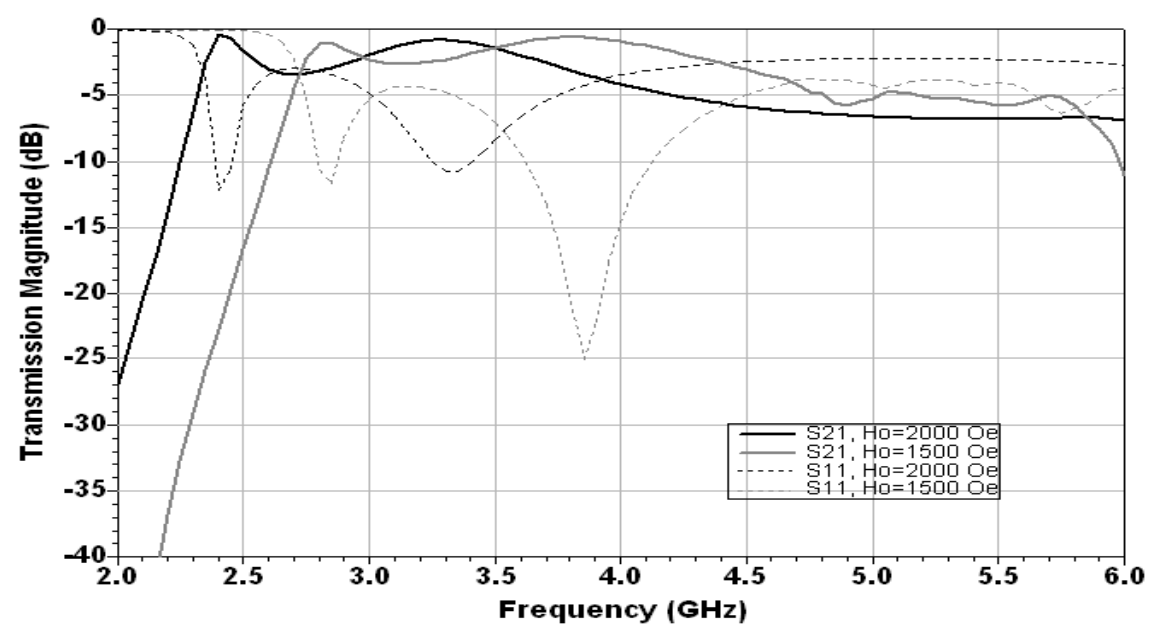

Figure 3. The full wave scattering parameters magnitude of the proposed transformer for $H_{0}=1500$ and 2000 Oe. 


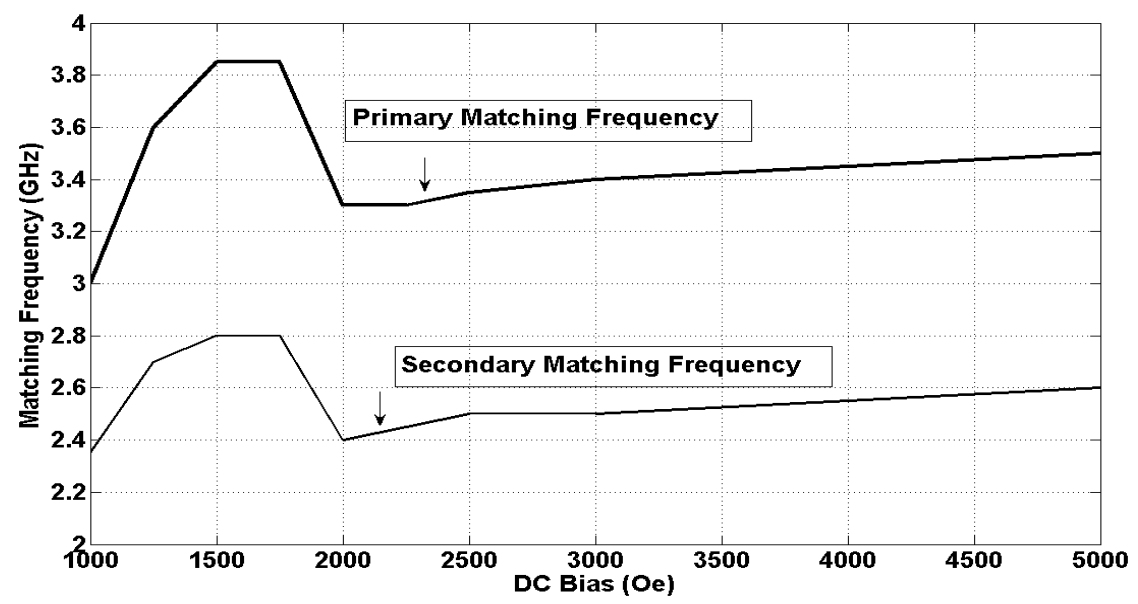

Figure 4. The change of the primary and secondary matching frequencies against the applied DC magnetic Bias.

\section{CONCLUSIONS}

A compact and tunable LH quarter wave transformer has been presented. The proposed transformer has been designed in CPW configuration on ferrite substrate. The proposed transformer has tunable performance with two operating bands over the frequency bandwidth from $2.35 \mathrm{GHz}$ to $3.85 \mathrm{GHz}$ and with return loss close to $20 \mathrm{~dB}$. The transformer has been studied analytically and verified numerically. The numerical results confirm the tuning capability of the proposed transformer by changing the applied DC magnetic bias.

\section{REFERENCES}

1. Veselago, V. G., "The electrodynamics of substances with simultaneously negative values of $\varepsilon$ and $\mu$," Soviet Physics Uspekhi, Vol. 10, 509-514, 1986.

2. Smith, R., W. J. Padilla, D. C. Vier, S. C. Nemat-Nasser, and S. Schultz, "Composite medium with simultaneously negative permeability and permittivity," Physical Review Lett., Vol. 84, 4184-4187, 2000.

3. Falcone, F., T. Lopetegi, J. D. Baena, R. Marques, F. Martin, and M. Sorolla, "Effective negative- $\varepsilon$ stopband microstrip lines based on complementary split ring resonators," IEEE Microwave and Wireless Components Lett., Vol. 14, No. 6, 280-282, 2004. 
4. Elefteriades, G. V., A. K. Iyer, and P. C. Kremer, "Planar negative refractive index media using planar L-C loaded transmission line," IEEE Trans. Microwave Theory \& Tech., Vol. 50, No. 12, 27022712, 2002.

5. Caloz, C. and T. Itoh, "Transmission line approach of left-handed (LH) materials and microstrip implementation of an artificial LH transmission line," IEEE Trans. Antennas and Propagation, Vol. 52, No. 5, 1159-1166, 2004.

6. Machae, J., M. Hudlieka, J. Zehentner, N. G. Spiliotis, and A. S. Omar, "Left-handed coplanar waveguide," Proc. IEEE MTT-S, 1957-1960, 2005.

7. Gao, J. and L. Zhu, "Guided-wave characteristics of CPW transmission line metamaterials: Effective per-unit-length parameters," Proceedings of IEEE International Workshop on Antenna Technology: Small Antennas and Novel Metamaterials, 175-178, March 7-9, 2005.

8. Caloz, C. and T. Itoh, Electromagnetic Metamaterials Transmission Line Theory and Microwave Applications, 1st edition, John Wiley \& Sons, New Jersey, 2006.

9. Eleftheriades, G. V. and K. G. Balmain, Negative Refractive Metamaterials, 1st edition, John Wiley \& Sons, New Jersey, 2005.

10. Lax, B. and K. J. Button, Microwave Ferrites and Ferrimagnetics, McGraw Hill, 1962.

11. Tsutsumi, M. and T. Ueda, "Nonreciprocal left-handed microstrip lines using ferrite substrate," Proceedings of IEEE MTT-S Int. Microwave Symposium, 249-252, USA, June 2004.

12. Tsutsumi, M. and T. Ueda, "Left handed transmission characteristics of ferrite microstrip lines without series capacitive load," IEICE Trans. Electron., Vol. E89, No. 9, 1318-1323, 2006.

13. Abdalla, M. and $\mathrm{Z}$. $\mathrm{Hu}$, "On the study of CWP dual band left handed propagation with reciprocal and nonreciprocal characteristics over ferrite substrates," Proceedings of IEEE AP-S Int. Symp., 2578-2581, USA, June 2007.

14. Abdalla, M. and Z. Hu, "Nonreciprocal left handed coplanar waveguide over ferrite substrate with only shunt inductive load," IEEE Microwave and Optical Technology Lett., Vol. 49, No. 11, 2810-2814, 2007.

15. Abdalla M. and Z. Hu, "On the study of left-handed coplanar waveguide coupler on ferrite substrate," Progress In Electromagnetics Research Letters, Vol. 1, 69-75, 2008. 